\title{
The influence of distinctive processing on retrieval-induced forgetting
}

\author{
REBEKAH E. SMITH and R. REED HUNT \\ University of North Carolina, Greensboro, North Carolina
}

\begin{abstract}
Recall of a portion of a previously experienced list benefits subsequent recall of that portion of the list but leads to poorer recall of nonpracticed items from the same set (Anderson, Bjork, \& Bjork, 1994). One explanation for this retrieval-induced forgetting is that during practice of part of a set, the nonpracticed items compete for recall and are suppressed; this suppression process inhibits later recall of the nonpracticed items. Two experiments were conducted to investigate the relationship between distinctive processing of the original set and retrieval-induced forgetting, on the assumption that distinctive processing reduces response competition. In the first experiment, distinctive processing induced by difference judgments among the studied items did reduce forgetting relative to a standard encoding task and a similarity judgment task. In fact, the difference judgment task completely eliminated retrievalinduced forgetting. In the second experiment, the similarity judgment task was analyzed in relation to a task assumed to foster associative integration (Anderson \& McCulloch,1999). Even though the similarity judgment met the requirements for associative integration, retrieval-induced forgetting persisted following similarity judgment. The results are consistent with the view that distinctive processing benefits memory within an organizational context (Hunt \& McDaniel, 1993; Smith \& Hunt, in press).
\end{abstract}

Anderson, Bjork, and Bjork (1994) discovered that recall of part of an episode impairs subsequent memory for the remainder of the episode, and this phenomenon, labeled retrieval-induced forgetting, has subsequently been pursued by Anderson and his colleagues (e.g., Anderson \& McCulloch, 1999; Anderson \& Spellman, 1995; Shaw, Bjork, \& Handal, 1995). The goal of the research presented here is to investigate the influence of organizational and distinctive processing on retrieval-induced forgetting. Specifically, we derive predictions concerning the elimination of retrieval-induced forgetting from the perspective of organizational/distinctive processing and report two experiments in which we tested those predictions.

Anderson and his colleagues (Anderson et al., 1994; Anderson \& Spellman, 1995) developed a three-phase paradigm to study retrieval-induced forgetting. In the first phase, participants study a list of words consisting of category labels and exemplars; for example, six instances of eight categories are presented. The second phase requires participants to practice retrieving three members of four of the categories. These practiced items are denoted Rp + items. The three members that were not practiced, but that belong to the four categories that did receive practice, are called $\mathrm{Rp}$ - items. Retrieval-induced forgetting is demonstrated during the third phase, a recall test for all

We are grateful to Michael Anderson, Robert Greene, and an anonymous reviewer for comments on a previous version of this paper, and to Michael Anderson for discussions about the second experiment reported in the current manuscript. Address correspondence concerning this article to Reed Hunt, University of North Carolina, Psychology Department, P.O. Box 26164, Greensboro, NC 27402-26164 (e-mail: huntrr@uncg.edu). of the original study items, if the following pattern of performance is produced: $\mathrm{Rp}+>\mathrm{Nrp}>\mathrm{Rp}-$. That is, items from partially practiced categories that have not themselves received practice are recalled at a rate below that of items from completely unpracticed categories.

Anderson and his colleagues attribute this forgetting to inhibition resulting from response competition (Anderson et al., 1994; Anderson \& Spellman, 1995). During the second phase of the paradigm when only half of the original list items are to be produced, other items, including list items, compete for recall. The competing items are presumed to be suppressed during this second phase, and the inhibition carries over to the final recall test for all of the first list items. Thus, the theory is that response competition results in inhibition of the nonpracticed $(\mathrm{Rp}-)$ list words. Consequently, eliminating response competition should eliminate retrieval-induced forgetting.

The paradigm for retrieval-induced forgetting enhances the probability of response competition during retrieval practice through the use of categorized lists. Categorized lists encourage the processing of similarity among the items, usually in the form of the categorical relationship. This relational processing is not optimal for memory because of difficulty in differentiating particular items at retrieval - essentially, the problem of response competition. Item-specific processing is the processing of differences among the items and is assumed to facilitate a discriminative process in retrieval that we call distinctiveness (Hunt \& McDaniel, 1993). Distinctive processing benefits retrieval by directing subjects to consider information that is specific to a particular target. Processing differences among elements of an event allows differentiation of elements that are otherwise similar along some dimension. 
When the differences are combined with the similarity, the resulting information is specific to a given item and facilitates retrieval (e.g., Hunt \& Einstein, 1981; Hunt \& Smith, 1996). Therefore, our hypothesis is that distinctive processing of the original study items will eliminate retrievalinduced forgetting presumably by reducing response competition in retrieval practice.

\section{EXPERIMENT 1}

The foregoing analysis of response competition implies that interference should be diminished when target items are distinctively processed. In our previous work, we have manipulated distinctive processing in several different ways, but perhaps the most straightforward of those has been the combination of list structure and orienting tasks at study. For example, we assume that a categorized list encourages the processing of similarity in the form of the shared category information. This organizational processing is important for item memory but not sufficient. In addition to the similarity among the items, the processing of differences is necessary to differentiate the specific items at retrieval.

In the first experiment, we used a very powerful method of inducing the processing of differences. This method combines the technique of self-generated cues (Mäntylä, 1986) with an explicit judgment of difference among the target items. An example of the power of this method can be found in Hunt and Smith (1996; Smith \& Hunt, in press), who asked subjects to study lists of categorized words by producing one thing that they knew about each word that was not also true of any of the other items. On a subsequent surprise memory test, subjects who received their own cues from study exhibited nearly perfect recall. Importantly for the present purposes, subjects who generated difference cues at study and were later given category label cues at test performed much better than subjects who generated shared cues (usually category labels) at study and were given those same labels as cues.

Thus, in the first experiment, subjects were asked to generate either differences or similarities among the target items. Both cases required self-generation, but only the production of differences was expected to reduce or eliminate response competition in the second phase of the retrieval-induced forgetting paradigm. We predicted that generation of differences among the target items would reduce retrieval-induced forgetting relative to generation of similarities and to the standard paired associate condition.

\section{Method}

\section{Subjects and Design}

Forty-eight volunteers participated in this study for credit in an introductory psychology course. Participants were randomly assigned to one of three groups reflecting the three levels of the between-subjects manipulation of encoding condition: standard paired associate learning, difference judgment, or similarity judgment. The second variable was item type, and each participant ex- perienced all three levels of this variable. As described in the introduction, $\mathrm{Rp}+$ items were those that received retrieval practice; $\mathrm{Rp}-$ items were from the same categories as $\mathrm{Rp}+\mathrm{items}$, but did not receive retrieval practice; and Nrp items were from categories for which no retrieval practice occurred for any item.

\section{Materials}

The materials used in this experiment were identical to those used by Anderson et al. (1994). Rather than reiterate the lengthy description of the item selection process and particular item ordering procedures, we refer readers to the original source for a detailed description. We shall provide a more general description, including any deviation from the procedure of Anderson et al.

The 60 items presented for study consisted of 6 instances from each of eight categories plus 12 filler items. The critical items were the high ranking category exemplars used by Anderson et al. (1994) in their third experiment (see p. 1087 of Anderson et al. for the specific items). The 12 filler items were 6 instances from each of two categories that also were used in the Anderson et al. study. Two separate study list orders were used.

For the retrieval practice phase, four lists were constructed so that each of the eight test categories served equally often as a practiced and as a nonpracticed category. Furthermore, each item served as a practiced and as a nonpracticed member of a practiced category equally often. Finally, category labels were provided as cues in the final test, and the labels were presented randomly for each subject, with the exception that one filler category label was always displayed first followed by the labels corresponding to the eight target categories. The experiment was conducted on personal computers controlled by a program written with the Micro Experimental Laboratory package (Schneider, 1988).

\section{Procedure}

Study phase. The subjects in the standard paired associate condition saw the category label displayed with each category member for $5 \mathrm{sec}$ and were instructed to spend the $5 \mathrm{sec}$ relating the items and the category label. Presentation conditions differed in the other two conditions in that the six category instances were presented simultaneously and the label was not present. The subjects were instructed to type one word describing how the top item in the block of six instances was either similar to or different from the other five instances in the block.

The list was shown six times, and the spatial arrangement of the items within a category differed on each presentation in such a way that each of the six instances served as the top item on one of the presentations. The subjects in the difference judgment condition typed one word for each target that described what was different about that target from the other five words in the block. The subjects in the similarity judgment condition typed one word for each target that described what was the same about that target and the other five words in the block. Both judgment tasks were subject-paced.

Retrieval practice phase. Retrieval practice consisted of completing a word stem with a word that was shown during the study phase. Each stem was shown along with a category label, and subjects were instructed to complete each stem with a word from the study list that began with those two letters. Each stem and label combination was shown on three different spaced retrieval trials. The category label and stem were displayed on the computer monitor for $10 \mathrm{sec}$, and subjects typed in their responses on the keyboard.

Filler task. Subjects performed a prospective memory task embedded in a lexical decision task. None of the target words from the retrieval-induced forgetting appeared in this task, which lasted approximately $20 \mathrm{~min}$.

Final recall test. The category label cues for the final test were presented in a random order, and subjects were allowed $1 \mathrm{~min}$ to respond to each cue. 
Table 1

Experiment 1: Mean Proportion of Words Recalled

(as a Function of Study Task and Item Type) and Mean

Retrieval-Induced Forgetting (as a Function of Study Task)

\begin{tabular}{|c|c|c|c|c|c|c|c|c|}
\hline \multirow[b]{3}{*}{ Study Task } & \multicolumn{6}{|c|}{ Item Type } & \multirow{2}{*}{\multicolumn{2}{|c|}{ 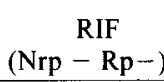 }} \\
\hline & \multicolumn{2}{|c|}{$\mathrm{Rp}+$} & \multicolumn{2}{|c|}{ Nrp } & \multicolumn{2}{|c|}{$\mathrm{Rp}-$} & & \\
\hline & $M$ & $S D$ & $M$ & $S D$ & $M$ & $S D$ & $M$ & $S D$ \\
\hline Diffe & .90 & .09 & .82 & .1 & .8 & .11 & -.03 & .11 \\
\hline Standa & .78 & .20 & .49 & .14 & .3 & .22 & .15 & .20 \\
\hline Similarity judgment & .81 & .10 & .51 & .10 & .38 & .21 & .14 & .15 \\
\hline
\end{tabular}

Note - RIF, retrieval-induced forgetting $=\mathrm{Nrp}-\mathrm{Rp}-$.

\section{Results}

\section{Retrieval Practice}

The mean proportions of stems completed during the retrieval practice task following the difference judgment, $M=.92$, similarity judgment, $M=.84$, and standard encoding conditions, $M=.88$, were subjected to an analysis of variance, which showed no main effect of study type on the proportion of stems completed during the retrieval practice task $\left[F(2,45)=2.11, M S_{\mathrm{e}}=.01\right]$.

\section{Final Test Performance}

The mean proportions of items recalled are presented in Table 1 as a function of item type and study task. Study task reliably affected overall recall $[F(2,45)=42.58$, $\left.M S_{\mathrm{e}}=.04\right]$, as did item type $\left[F(2,90)=75.61, M S_{\mathrm{e}}=.02\right]$. These effects, however, were qualified by a significant interaction of the two variables $\left[F(4,90)=12.79, M S_{\mathrm{e}}=\right.$ $.02]$. The important constituent of this interaction was the lack of retrieval-induced forgetting in the difference judgment condition. Separate analysis of variance for each study task condition showed significant effects of item type on recall following similarity judgment $[F(2,30)=$ $\left.46.27, M S_{\mathrm{e}}=.02\right]$, and standard encoding $[F(2,30)=$ $\left.35.07, M S_{\mathrm{e}}=.02\right]$. The influence of item type approached, but did not reach significance following the difference judgment $\left[F(2,30)=3.29, M S_{\mathrm{e}}=.01, p=.05\right]$. Whereas significant forgetting occurred in the similarity judgment $[t(15)=3.70]$ and standard encoding conditions $[t(15)=$ 3.13] conditions, a paired samples $t$ test demonstrated that $\mathrm{Rp}-$ and Nrp item types did not differ for the subjects in the difference judgment condition $[t(15)=1.18]$.

Finally, individual comparisons of study tasks on Nrp items showed reliable differences between the difference judgment task and the standard condition $[t(30)=7.47]$ and between the difference judgment and the similarity judgment $[t(30)=8.10]$. The similarity judgment and standard encoding conditions did not differ with respect to recall of the Nrp items $[t(30)=.54]$.

\section{DISCUSSION}

The results of the first experiment clearly demonstrate the elimination of retrieval-induced forgetting following an orienting task that required detection of differences among the target items. Difference judgment at study was followed by statistically equivalent recall of Nrp and $\mathrm{Rp}$ - items, whereas an orienting task requiring similarity judgment and the standard paired associate task both continued to yield poorer recall of $\mathrm{Rp}-$ items relative to Nrp items.

There are, however, several concerns about the interpretation of the data. The first issue is the possibility of ceiling effects on recall following difference judgments, which would obscure potential differences between Nrp and $\mathrm{Rp}-$ items. Given the reliable difference between $\mathrm{Rp}+$ and Nrp items $[t(15)=2.13]$, this concern may not be critical. The second point involves the procedural modification of repeating items at study, a modification required in order to obtain the similarity and difference judgments. Given that the difference judgment eliminated retrieval-induced forgetting but the similarity judgment did not, repetition alone was not responsible for the critical outcome because the same amount of repetition occurred in the two conditions. Furthermore, the fact that six repetitions of the similarity condition produced recall equivalent to that for one presentation of the standard condition essentially replicates data from Hunt and Einstein (1981). Hunt and Einstein showed that repetition of an orienting activity directed to a dimension congruent with the dominant list structure had no effect; that is, two similarity judgments on a categorized list were no better than one, and two judgments of difference on an unrelated list were no better than one.

Nonetheless, recent work of Anderson and McCulloch (1999) suggests an alternative account of our results. In their experiments, subjects were asked to study the items by thinking of the other items that had been presented in that category. The theoretical purpose of this manipulation was to establish an associative integration among the instances within each category. These instructions to integrate items reduced retrieval-induced forgetting relative to the standard instruction condition. Perhaps our difference judgment study task eliminated retrieval-induced forgetting because of associative integration rather than because of distinctive processing. The difference judgment requires consideration of the relationship among the instances of the category in much the same way that instructions to think of other items in the category might. On the other hand, our similarity judgment task also asked 
subjects to consider the relationship among the items of a category, but it is possible that the similarity task only required superficial processing of inter-instance relationships. Furthermore, because the instances were presented in a simultaneous block, each of which were repeated six times, subjects may have done little processing of the instances beyond recognizing which category was present. The point is that integration, not distinctiveness, may be the reason for the elimination of retrieval-induced forgetting.

The strength of this argument rests on what is happening in the similarity judgment condition. If the similarity judgment does involve comparison among the instances, then the conditions are set for integration, and retrievalinduced forgetting should be eliminated. On the other hand, if subjects are explicitly encouraged to relate the items in the similarity condition and retrieval-induced forgetting still occurs, one might conclude that associative integration of the items is less important than reduction of similarity among the items. We conducted a second experiment designed to encourage interitem comparisons in the similarity judgment condition.

\section{EXPERIMENT 2}

The similarity judgments in the first experiment were made on blocks of items presented simultaneously. The concern about this procedure is that subjects could make their judgment without detailed consideration of the relationship among the items. Given the strong categorical relationship among items, subjects could provide a category label as the dimension of similarity with only cursory examination of the items. Moreover, because the similarity judgment occurred six times over the same items, judgments subsequent to the first presentation could have been made by only examining the first item in the block. The implication is that little interitem integration may be fostered by this procedure.

We corrected this problem in Experiment 2 by giving subjects in the similarity judgment condition the same instructions to integrate as were used by Anderson and McCulloch (1999). On presentation of an item, Anderson and McCulloch's instructions were to think about all of the other items that had been presented in the category. We used the same instructions, combined with encouragement to think about how the items were similar. Furthermore, unlike Anderson and $\mathrm{McCulloch}$, we asked subjects to provide the dimension of similarity each time an item was presented, allowing an objective measure of what the subjects were doing.

The similarity condition was contrasted to the standard paired associate procedure and to Anderson and $\mathrm{McCul}-$ loch's (1999) integration condition. If, as suggested by Anderson and McColloch, the instructions to think about all of the items previously presented in a category results in associative integration which in turn eliminates retrievalinduced forgetting, then our similarity judgment condition should mimic the integration condition. If, on the other hand, the critical factor in reducing retrieval-induced forgetting is the reduction of similarity among the items, we should continue to see retrieval-induced forgetting in the similarity judgment condition in spite of the instructions to integrate items.

\section{Method \\ Subjects and Design \\ Forty-eight volunteers participated in this study for credit in an introductory psychology course. The subjects were randomly as- signed to one of three groups reflecting the three levels of the between-subjects manipulation of encoding condition: standard paired associate learning, similarity judgment, or integration. The second variable was item type, which was the same as in Experi- ment 1 , and each subject experienced all three levels of this variable.}

\section{Materials \\ The materials and equipment match those of Experiment 1.}

\section{Procedure}

Study phase. The standard encoding condition was the same as in Experiment 1: category label and member pairs were presented for $5 \mathrm{sec}$ each and the subjects related the item and the category label. The integration condition was the same as the standard encoding condition, except that subjects had to try to remember the previously presented members of a category. The similarity judgment condition was the same as the integration condition with the additional task of typing in one word that described something that was the same about the current category member and all of the previously presented category members. The subjects did not type in a word for the first presentation from a particular category.

Retrieval practice phase. The retrieval practice phase was the same as the retrieval practice phase in Experiment 1.

Filler task. Subjects performed a prospective memory task embedded in a lexical decision task. None of the target words from the retrieval-induced forgetting appeared in this task, which lasted approximately $20 \mathrm{~min}$.

Final recall test. The procedures for the final test phase remained unchanged from Experiment 1.

\section{Results and Discussion}

\section{Retrieval Practice}

The mean proportions of stems completed during the retrieval practice phase following standard encoding, $M=.86$, similarity judgment, $M=.87$, and integration, $M=.83$, did not differ as a function of study task $(F<1)$.

\section{Final Recall Test Performance}

The mean proportions of words recalled are reported in Table 2 as a function of item type and study condition. An analysis of variance produced a main effect of item type $\left[F(2,90)=124.87, M S_{\mathrm{e}}=.02\right]$, but no main effect of study task and no interaction of the two variables $(F \mathrm{~s}<1)$. In order to evaluate our predictions concerning the differential impact of the similarity judgment task and integration instructions on retrieval-induced forgetting, planned comparisons for Nrp and Rp - item recall were conducted for each study task condition. As expected, the standard encoding instructions produced significant forgetting on the final recall test $[t(15)=1.96]$, whereas in- 
Table 2

Experiment 2: Mean Proportion of Words Recalled

(as a Function of Study Task and Item Type) and Mean

Retrieval-Induced Forgetting (as a Function of Study Task)

\begin{tabular}{|c|c|c|c|c|c|c|c|c|}
\hline \multirow[b]{3}{*}{ Study Task } & \multicolumn{6}{|c|}{ Item Type } & \multirow{2}{*}{\multicolumn{2}{|c|}{$\begin{array}{c}\text { RIF } \\
(\mathrm{Nrp}-\mathrm{Rp}-)\end{array}$}} \\
\hline & \multicolumn{2}{|c|}{$\mathrm{Rp}^{+}$} & \multicolumn{2}{|c|}{$\mathrm{Nrp}$} & \multicolumn{2}{|c|}{$\mathrm{Rp}-$} & & \\
\hline & $M$ & $S D$ & $M$ & $S D$ & $M$ & $S D$ & $M$ & $\overline{S D}$ \\
\hline Similarity & .74 & .16 & .46 & .13 & 36 & .15 & .10 & .13 \\
\hline Standard encoding & .74 & .16 & .40 & .11 & 32 & .16 & .08 & .16 \\
\hline Integration & .69 & .17 & .40 & .13 & .37 & .13 & .03 & .16 \\
\hline
\end{tabular}

Note-RIF, retrieval-induced forgetting $=\mathrm{Nrp}-\mathrm{Rp}-$.

tegration instructions did not $[t(15)=.45]$. Consistent with our prediction, the similarity judgment task led to significant forgetting $[t(15)=3.24]$.

\section{Cue Analysis}

To determine whether subjects in the similarity judgment condition were making perfunctory judgments of the items, we examined the cues produced by each subject in this condition. For each subject, the proportion of different cues produced within each category was computed. This datum speaks to the question of whether subjects are simply producing the category label when an instance is provided, an outcome that would be consistent with the notion that the judgment is based on a superficial processing of the items rather than more extensive consideration that might be consistent with integration. The mean proportion of unique cues was .53. Thus, half the cues for each category were different, indicating that subjects were not making exactly the same similarity judgment for each word. Moreover, when the proportion of unique cues was entered as a regression variable for an analysis of the amount of retrieval-induced forgetting, the impact was insignificant $(F<1)$. As yet a further step, we divided the subjects into two groups based on whether the number of different cues was above or below the mean. Analysis of retrieval-induced forgetting as a function of group was not reliable $[t(14)=.15]$.

Instructions to integrate the items within a category had different effects on retrieval-induced forgetting, depending on whether those instructions included reference to similarity. When the instructions were the same as those of Anderson and McCulloch (1999) to think about the other items in the category, retrieval-induced forgetting was eliminated, replicating their finding. However, when exactly the same instructions were appended with the instruction to think about similarity among the items, retrieval-induced forgetting was of comparable magnitude to that for the standard presentation procedure.

\section{GENERAL DISCUSSION}

In the experiments reported here, we examined predictions about retrieval-induced forgetting from an organizational/distinctive view of memory. The paradigm for retrieval-induced forgetting maximizes the probabil- ity of interference because of the categorical similarity of the to-be-remembered items. Previous research has demonstrated that categorically related items are remembered much better when subjected to some form of differential processing at study (e.g., Begg, 1978; Hunt \& Einstein, 1981; Hunt \& Smith, 1996). Thus, at a general level, one might expect that variables encouraging differential processing at study would enhance performance in the retrieval-induced forgetting paradigm. Our first experiment yielded results consistent with this assumption by showing that subjects asked to detect differences among the study items did not suffer retrieval-induced forgetting. Study tasks requiring either the detection of similarities among the items or intentional memory did produce reliable retrieval-induced forgetting.

We assume that the encoding processes encouraged by the difference judgment task facilitate discrimination among the otherwise similar items by providing a basis for individual item differentiation. The combined processing of similarity and difference sharpens memory for the individual items (Hunt \& McDaniel, 1993; Hunt \& Smith, 1996; Smith \& Hunt, in press), and on the presumption that response competition in retrieval practice is a necessary precursor to retrieval-induced forgetting (Anderson et al., 1994; Anderson \& Spellman, 1995), the combined processing of organization and distinctiveness reduces response competition.

An alternative interpretation of the effects of the difference judgment task is available from the work of Anderson and McCulloch (1999). On this view, the effect of difference judgment is to establish, or more likely strengthen, the associations among the study items. This process, called integration, is presumed to result from comparing the items at study. Anderson and McCulloch have demonstrated that instructions to think of all of previously presented instances of a given category on presentation of an instance eliminates retrieval-induced forgetting. Perhaps the item comparisons required in order to make a difference judgment task enhance associative integration.

If so, however, one wonders why the similarity judgment continued to yield retrieval-induced forgetting. On the face of it, similarity judgment also requires comparisons among items. One possibility is that the similarity judgment condition of the first experiment actually did not require subjects to compare the items to render their 
judgment. In the second experiment, we addressed this possibility by explicitly instructing the subjects to integrate the items in the course of making their similarity judgment. The subjects in the second experiment continued to show retrieval-induced forgetting in the similarity judgment condition.

The results of the second experiment bear strongly on the interpretation of why difference judgments eliminated retrieval-induced forgetting in Experiment 1. On Anderson and McCulloch's (1999) interpretation, one might assume that difference judgments resulted in associative integration, but if so, one then must account for the fact that similarity judgments did not also eliminate retrievalinduced forgetting in that similarity judgment required consideration of all of the items. A possible reason for this failure is that the similarity judgments did not require consideration of all of the items and thus integration did not occur. Experiment 2 eliminated this possibility.

The very same instructions that Anderson and $\mathrm{McCul}$ loch (1999) used to encourage the formation of associative relationships were given to the similarity group in Experiment 2, yet that group's performance closely resembled that in the similarity judgment condition in Experiment 1 . Moreover, the similarity judgments made by the subjects in Experiment 2 showed considerable variability across judgment opportunities. This datum goes to the criticism of Experiment 1 that the similarity judgment could be made with superficial processing of the items, resulting in a perfunctory judgment corresponding to the category label. Clearly the subjects in Experiment 2 were not doing this, and the judgments rendered are consistent with the assumption that the subjects were considering previous items on each judgment opportunity. Furthermore, no relationship existed between the number of different similarity judgments and the amount of retrieval-induced forgetting. Thus, the results of the second experiment are consistent with the idea that the qualitative nature of the judgment is the critical factor for reducing retrieval-induced forgetting.

In summary, retrieval-induced forgetting is a reliable phenomenon that has considerable implications for applied issues in memory. Consequently, the theoretical mechanisms underlying retrieval-induced forgetting are of some importance. Anderson et al. (1994) have convincingly argued against an account based on associative strength dependent response competition in the final test. In its stead, they hypothesize that response competition at retrieval practice produces inhibition of nonpracticed items. Our data are not inconsistent with the inhibitory account, on the assumption that the combined encoding of organizational and distinctive information reduces response competition in retrieval practice; but our experiments were not designed to directly address the mechanisms of retrieval-induced forgetting. What our data do demonstrate is that the occurrence of retrievalinduced forgetting is bounded by the processing of similarity and difference at encoding of the original experience. This boundary should be of some use in constraining a theory of retrieval-induced forgetting.

\section{REFERENCES}

Anderson, M. C., BJork, R. A., \& Bjork, E. L. (1994). Remembering can cause forgetting: Retrieval dynamics in long-term memory. Journal of Experimental Psychology: Learning, Memory, \& Cognition, 20, 1063-1087.

Anderson, M. C., \& MCCulloch, K. C. (1999). Integration as a general boundary condition on retrieval-induced forgetting. Journal of Experimental Psychology: Learning, Memory, \& Cognition, 25, 608-629.

Anderson, M. C, \& Spellman, B. A. (1995). On the status of inhibitory mechanisms in cognition: Memory retrieval as a model case. Psychological Review, 102, 68-100.

BEGG, I. (1978). Similarity and contrast in memory for relations. Memory \& Cognition, 6, 509-517.

HunT, R. R., \& EINSTEIN, G. O. (1981). Relational and item-specific information in memory. Journal of Verbal Learning \& Verbal Behavior, 20, 497-514.

Hunt, R. R., \& MCDANiEL, M. A. (1993). The enigma of organization and distinctiveness. Journal of Memory \& Language, 32, 421-445.

Hunt, R. R., \& SMITH, R. E. (1996). Accessing the particular from the general: The power of distinctiveness in the context of organization. Memory \& Cognition, 24, 217-225.

MäNTYLÄ, T. (1986). Optimizing cue effectiveness: Recall of 500 and 600 incidentally learned words. Journal of Experimental Psychology: Learning, Memory, \& Cognition, 12, 66-71.

SCHNEIDER, W. (1988). Micro Experimental Laboratory: An integrated system for IBM PC compatibles. Behavior Research Methods, Instruments, \& Computers, 20, 206-217.

Shaw, J. S., III, BJorK, R. A., \& HANDAL, A. (1995). Retrieval-induced forgetting in an eyewitness-memory paradigm. Psychonomic Bulletin \& Review, 2, 249-253.

Smith, R. E., \& Hunt, R. R. (in press). The effects of distinctiveness require reinstatement of organization: The importance of intentional memory instructions. Journal of Memory \& Language.

(Manuscript received January 20, 1998; revision accepted for publication July 22, 1999.) 